\title{
Burst Noise in the HAWAII-1RG Multiplexer
}

\author{
Candice M. Bacon ${ }^{a}$, Craig W. McMurtry ${ }^{a}$, Judith L. Pipher ${ }^{a}$, William J. Forrest ${ }^{a}$, \\ James D. Garnett ${ }^{b}$ \\ ${ }^{a}$ University of Rochester, Rochester, NY, USA \\ ${ }^{b}$ Rockwell Scientific Company, Camarillo, CA, USA
}

\begin{abstract}
Burst noise (also known as popcorn noise and random telegraph signal/noise) is a phenomenon that is understood to be a result of defects in the vicinity of a p-n junction. It is characterized by rapid level shifts in both positive and negative directions and can have varying magnitudes. This noise has been seen in both HAWAII-1RG and HAWAII-2RG multiplexers and is under investigation. We have done extensive burst noise testing on a HAWAII-1RG multiplexer, where we have determined a significant percentage of pixels exhibit the phenomenon. In addition, the prevalence of small magnitude transitions make sensitivity of detection the main limiting factor. Since this is a noise source for the HAWAII-1RG multiplexer, its elimination would make the HAWAII-1RG and the HAWAII-2RG even lower noise multiplexers.
\end{abstract}

Keywords: burst noise, popcorn noise, HAWAII-1RG, multiplexer noise, RTS, RTN, telegraph noise

\section{INTRODUCTION}

The HAWAII-1RG multiplexer was used in the development of $10 \mu \mathrm{m}$ cutoff, low dark current HgCdTe detector arrays for passively cooled $(30 \mathrm{~K})$ space-based astronomy missions. We chose a $36 \mu \mathrm{m}$ pixel pitch, which corresponds to the bonding of the $\mathrm{HgCdTe}$ pixels to every other column and row of the multiplexer. Thus, our clocking program is designed to read every other column and row, leading to an array size of $512 \times 512$ pixels, including 4088 reference pixels around the perimeter of the array. The pixels read by our clocking program are the focus of the tests that follow. We have run preliminary testing on all three arrays we received for this project, and have found similar results for all three. The most extensive tests were done on one of these arrays (H1RG-16-001), which will be the focus of this paper.

We first encountered burst noise while refining the algorithm we created to characterize dark current data from devices for our program. With data taken in sample up the ramp (SUTR) mode, we expect that the slope of the data will be continuous with the exception of cosmic ray hits. During the evaluation of individual pixels we noticed that the slope of the data was not always continuous. The data of some pixels exhibited what appeared to be positive and negative cosmic ray hits with varying magnitudes. An investigation revealed the source of this phenomenon was not cosmic rays at all, but rather burst noise. Burst noise can also be indicated by unusually noisy pixels. A typical pixel has noise corresponding to a $\sigma$ about the mean of $32 \mu$ Volts (input referred). However, some pixels have uncharacteristically larger standard deviations than that of a typical pixel. The extra noise in these pixels can be due to burst noise. For example, if a pixel has burst noise with transitions smaller than our detection limit, the pixel will merely appear to be slightly noisier than other pixels.

Burst noise, also known by many other names such as popcorn noise and RTS/RTN (Random Telegraph Signal/Noise), is a phenomenon that is understood to be a result of defects in the vicinity of a current carrying region. ${ }^{1-7}$ It is characterized by rapid current level shifts in both positive and negative directions and can have varying magnitudes. To cause burst noise, the generation or recombination in a single defect or trap either modifies the conductivity of a constrained current channel or reduces the energy barrier between two regions, enabling carriers to tunnel through the barrier more readily. Both of these lead to significant changes in current as a result of a single trapped or released charge. For an integrated circuit, such as the hybridized HAWAII-1RG

Further author information:

E-mail: candice@astro.pas.rochester.edu

Focal Plane Arrays for Space Telescopes II, edited by Thomas J. Grycewicz, Cheryl J. Marshall,

Proceedings of SPIE Vol. 5902 (SPIE, Bellingham, WA, 2005) · 0277-786X/05/\$15 - doi: 10.1117/12.624191

Proc. of SPIE 59020K-1 
multiplexers, a current change in any component in the signal chain from the detector diode of the unit cell to the signal output results in a change in voltage at the signal output.

Because this noise was seen both in forward and reverse detector bias, as well as in reference pixels (no detector), all of which were capable of producing very large magnitude transitions (on the order of $\mathrm{mV}$ ), the burst noise is attributed to the multiplexer. ${ }^{8}$ Since some pixels exhibit this noise while others do not, the origin of the noise must be within the unit cell of the multiplexer, making the most likely candidate the source follower unit cell MOSFET. Other authors ${ }^{9}$ have also detected burst noise in the HAWAII-1RG and HAWAII-2RG, but not, to our knowledge, in other multiplexers. The reason for this may be due to the $0.25 \mu \mathrm{m}$ design rules employed by Rockwell Scientific in manufacturing.

Many authors have reported observing burst noise in small dimension MOSFETs. ${ }^{2,5-7,10}$ In these reports, authors concentrate almost exclusively on two-level burst noise (sometimes referred to as a bistable waveform), indicating a single mechanism for the majority of devices exhibiting the phenomenon. To further investigate this noise source, assuming the source follower unit cell FET was the origin, a testing method was devised to prevent the $\mathrm{HgCdTe}$ diodes from contributing to the noise.

\section{TESTING FOR BURST NOISE}

To test for burst noise in the HAWAII-1RG multiplexer, the following procedure was used. First, the reset clock is always on, causing $V_{\text {reset }}$ to be applied to every pixel during each read throughout the entire integration. Also, the bias across the detector is set to zero $\left(V_{\text {reset }}=0\right.$ and $\left.D_{\text {sub }}=0\right)$. This eliminates the effects of the $\operatorname{HgCdTe}$ diode on the output (such as dark current). Other global voltage fluctuations are subtracted off with a single frame average of the reference pixels, leaving only the fluctuations due to the FETs within the unit cell.

The data are taken in SUTR mode, 3000 samples per integration, each sample two seconds apart (which is near the minimum frame read time at $100 \mathrm{kHz}$ operation). The integration is thus 6000 seconds long. Over the hour and 40 minutes of each integration, peculiar fluctuations are seen on some pixels, but not others. Samples of these fluctuations are shown in Section 3.

Integrations were obtained at three temperatures, $30 \mathrm{~K}, 37 \mathrm{~K}$, and $77 \mathrm{~K}$. These data were reduced using an IDL (Interactive Data Language) program that we wrote to detect burst noise. For a pixel without burst noise, it is expected that the average signal level (with respect to the global signal level) does not change over the entire integration. This is in fact what well behaved pixels show. Figure 1 shows the signal as a function of sample

Figure 1. Signal vs. sample number and corresponding histogram for a pixel without burst noise.
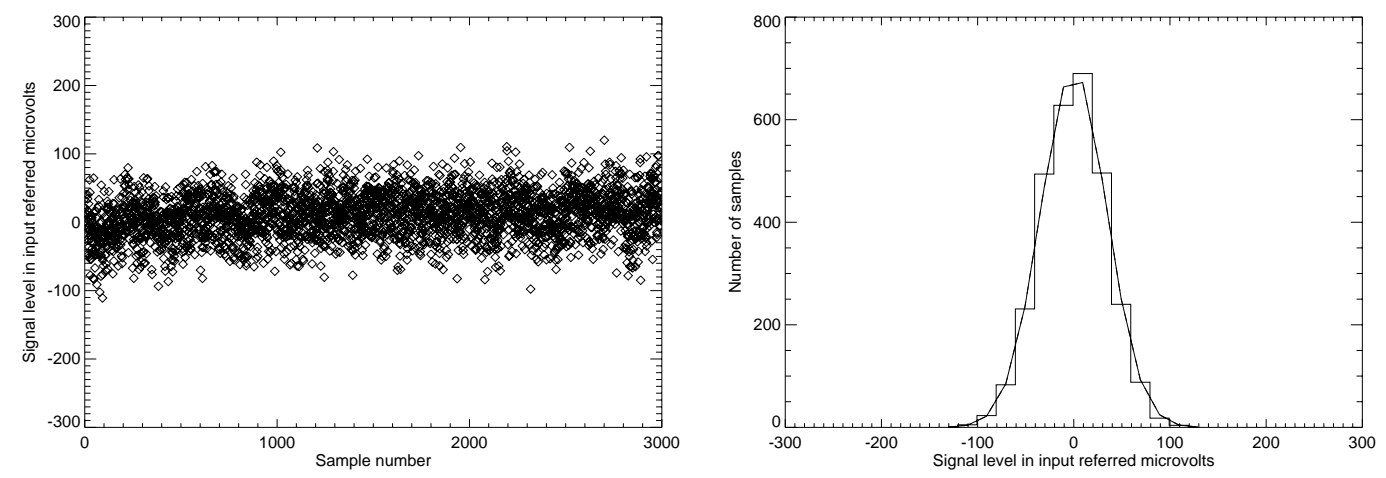

number and the corresponding histogram for a pixel that does not have burst noise. This figure and all following figures are for data taken at 30K, though similar figures can be found for each of the temperatures at which data were taken. The signal is shown in input referred microvolts, and the peak of the Gaussian distribution is centered at zero. (The average value of the dominant level is subtracted from the data to place its peak at zero.) 
In a pixel with burst noise, the average signal level over one period of time during the integration may not be the same as that over another, leading to the appearance of multiple levels. To detect these various levels, a histogram is plotted, indicating number of samples vs. signal level. A pixel without burst noise displays a single Gaussian around a single signal level. On the other hand, a pixel with burst noise displays multiple Gaussians, each around a different signal level. The detection of more than one Gaussian in a single histogram indicates a pixel that exhibits burst noise.

The minimum distinguishable separation between Gaussians determines the detectability of burst noise by this algorithm in pixels where parts of the multiple Gaussians overlap. To determine this separation, we used a modified version of the Rayleigh criterion for multiple Gaussians with varying sizes. In short, in order to distinguish between two Gaussians, the sum of the two individual Gaussians had to form a saddle point between them, and if more than one was fit concurrently, they were required to have the same standard deviation, since the standard devation represents the total pixel noise in the absence of burst noise. Also, the characteristic time for a given level may affect its detectability. If the levels overlap and a pixel spends equal time in each level, then the resultant histogram may indicate a single Gaussian. Therefore, the minimum resolution for a pixel depends on its total noise excluding burst noise in addition to the time spent in each level. Figure 2 shows a pixel with two very close levels which overlap. These close, nearly equally occupied levels result in a single Gaussian. Therefore, this pixel, though it has burst noise, is not detectable by our algorithm because it is below the threshold.

Figure 2. Signal vs. sample number and corresponding histogram for a pixel exhibiting two level burst noise below the threshold of detectability by the Rayleigh criterion in our algorithm. Note the width of the Gaussian in this histogram is slightly larger than that of Figure 1.
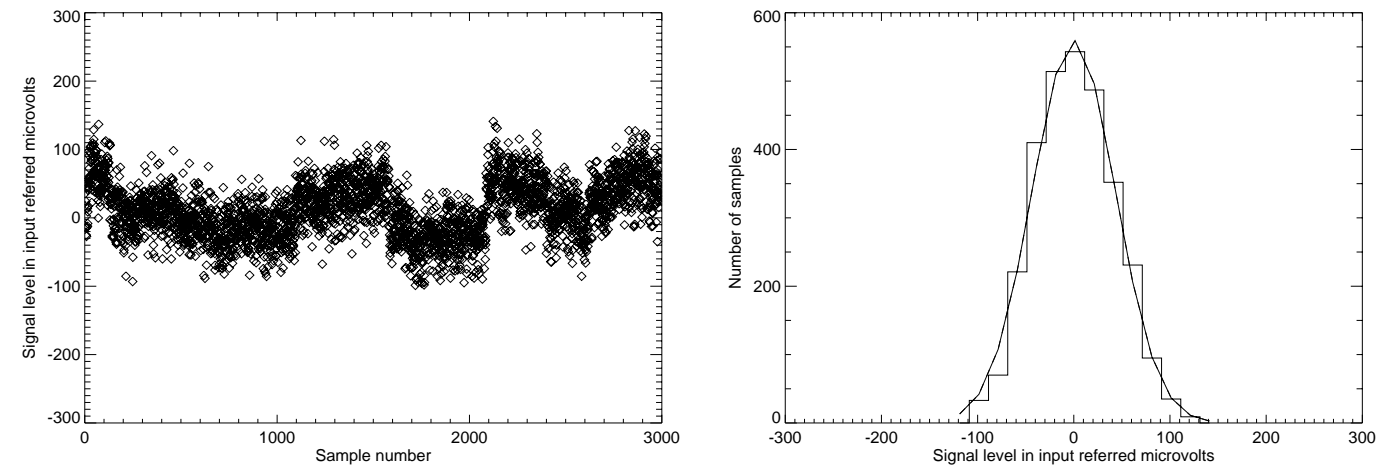

\section{TEMPORAL DEPENDENCE OF SIGNAL LEVEL}

For pixels whose signal level does change with respect to time, there are many variations in observed burst noise. Considering the various degrees of stability of the levels between transitions leads to a quantification of the observed behavior. The degree of stability is defined by the total time the pixel output remains in the same level before transitioning to another level. These levels can be stable (S), on average 1000 seconds or more, unstable (U), 10 seconds or less, or metastable (M), i.e. somewhere inbetween stable and unstable. For some pixels, the transitions happen so frequently, that it is unclear whether the pixel is actually remaining in the level in which it is measured, or whether the pixel level is merely undersampled, yielding an approximation of a much faster behavior. These levels will be considered unstable, reserving the term metastable for levels in which a pixel clearly remains for a period of time. While the two second frame read time limit influences the subjective determination of variation, this same limitation will be present whenever this multplexer is used with the same readout method. In fact, the minimum frame time will be greater (with $100 \mathrm{kHz}$ operation) if every pixel on the multiplexer is used. 
For two level burst noise, the most common case, the three degrees of stability (stable (S), metastable (M), and unstable $(\mathrm{U})$ ) make 6 unique variations. Table 1 details the possible variations. Each of these variations have been seen in HAWAII-1RG pixels, with some more common than others.

Table 1. Variations of dual level burst noise.

\begin{tabular}{ccc} 
Variation & Level 1 & Level 2 \\
\hline S-S & Stable & Stable \\
S-M & Stable & Metastable \\
S-U & Stable & Unstable \\
M-M & Metastable & Metastable \\
M-U & Metastable & Unstable \\
U-U & Unstable & Unstable
\end{tabular}

The U-U variation is apparent when the pixel may exhibit any level at any time. This variation can come about from undersampling as described above, and is the most common variation. One such pixel exhibiting this variation with two levels is shown in Figure 3. The dashed line shows the sum of the Gaussians. This particular pixel shows a preference for the lower level. Other pixels may show a preference for the upper level or may spend equal time in both levels. Notice that this pixel also has the preferred level centered at $0 \mu \mathrm{V}$ with approximately the same spread as Figure 1, but also has another distinct level where a Gaussian forms centered at $\sim 200 \mu \mathrm{V}$, leading to a 'peak to peak' separation of $\sim 200 \mu \mathrm{V}$.

Figure 3. Signal vs. sample number and corresponding histogram for a pixel exhibiting U-U burst noise. The dashed line shows the sum of the Gaussians.
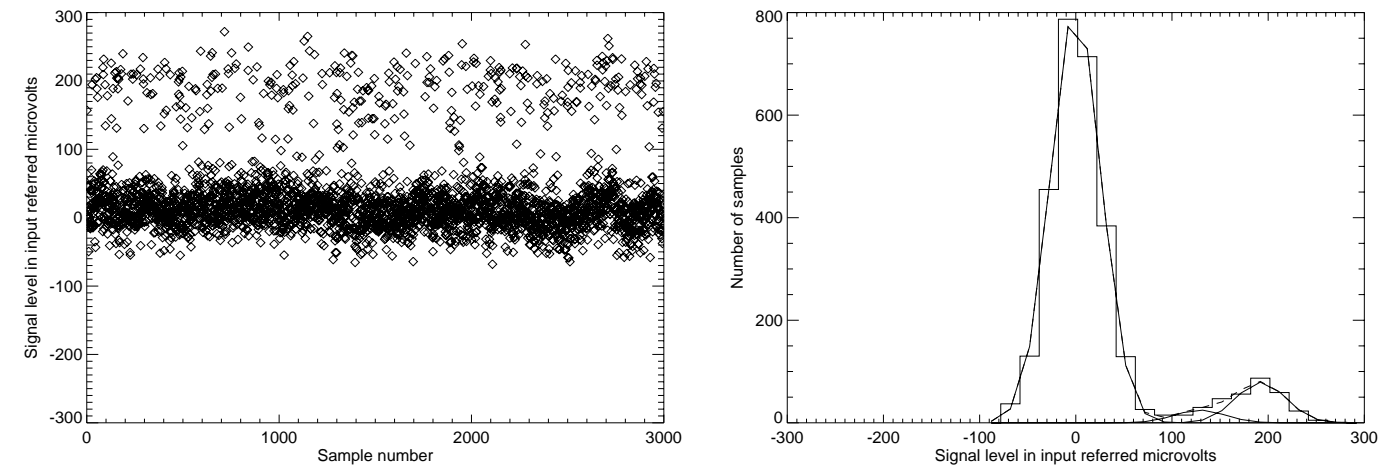

As the 'peak to peak' separation between neighboring Gaussians is decreased, it becomes harder to distinguish the burst noise with the eye, because the pixel merely appears noisier than usual. However, when the Gaussian fits are performed on the data, it becomes clear that the data are representative of more than one level relatively close together. One example of this proximity is shown in Figure 4. Here the 'peak to peak' separation is less than $100 \mu \mathrm{V}$.

When the frequency of transitioning to the secondary level decreases, the burst noise variation moves from U$\mathrm{U}$ to $\mathrm{M}-\mathrm{U}$, and eventually to S-U. In the same manner, the number of data points from a pixel in the secondary level decreases, making it increasingly more difficult to detect the secondary level when they are very close together. In addition, even levels greater than $4 \sigma$ apart can be difficult to detect when the unstable level has only a few data points, since more than one data point in a $20 \mu \mathrm{V}$ bin is required for a detection. Therefore, many pixels with close levels or very infrequent transitions that have M-U and S-U remain undetected by our burst noise detection algorithm. 
Figure 4. Signal vs. sample number and corresponding histogram for a pixel exhibiting U-U burst noise with levels relatively close together. The dashed line shows the sum of the Gaussians.
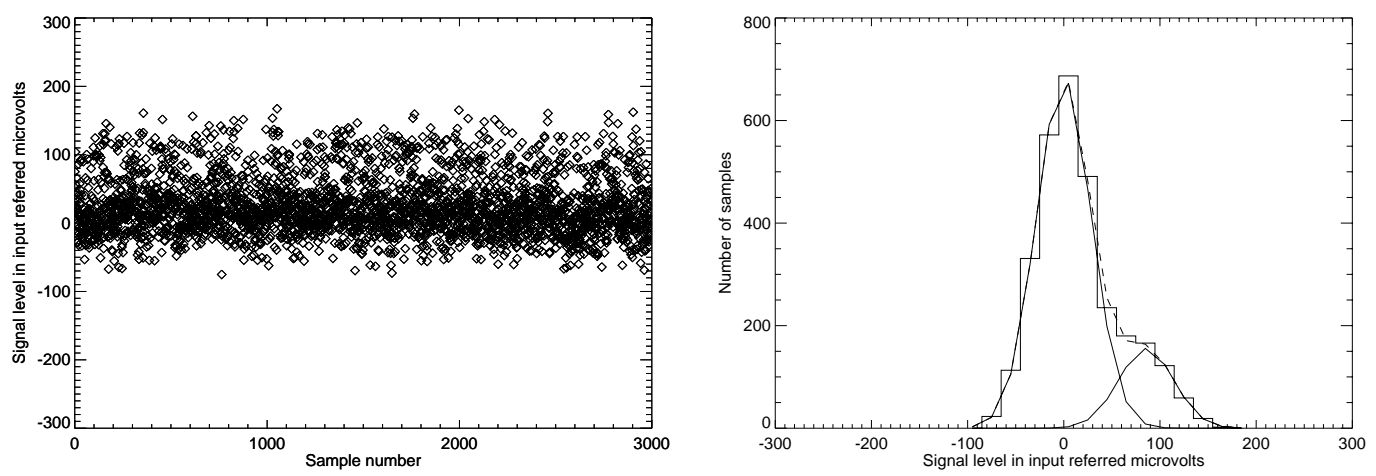

Figure 5. Signal vs. sample number and corresponding histogram for a pixel exhibiting M-U burst noise. Note the larger scale and indistinct secondary level(s).
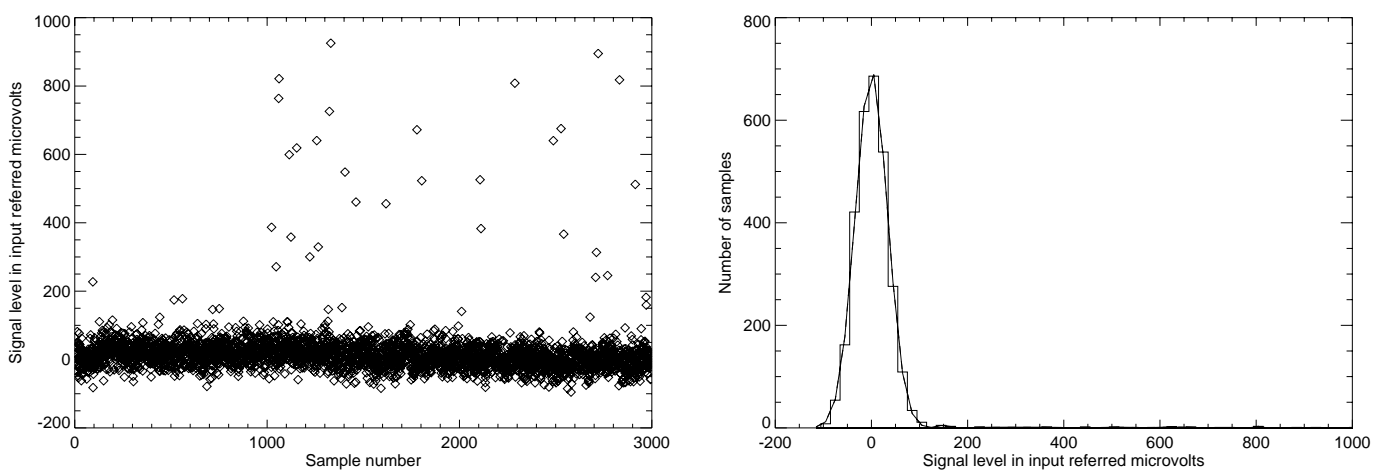

Figure 6. Signal vs. sample number and corresponding histogram for a pixel exhibiting S-U burst noise. Note the larger scale and detectable secondary level very close to the dominant signal level.
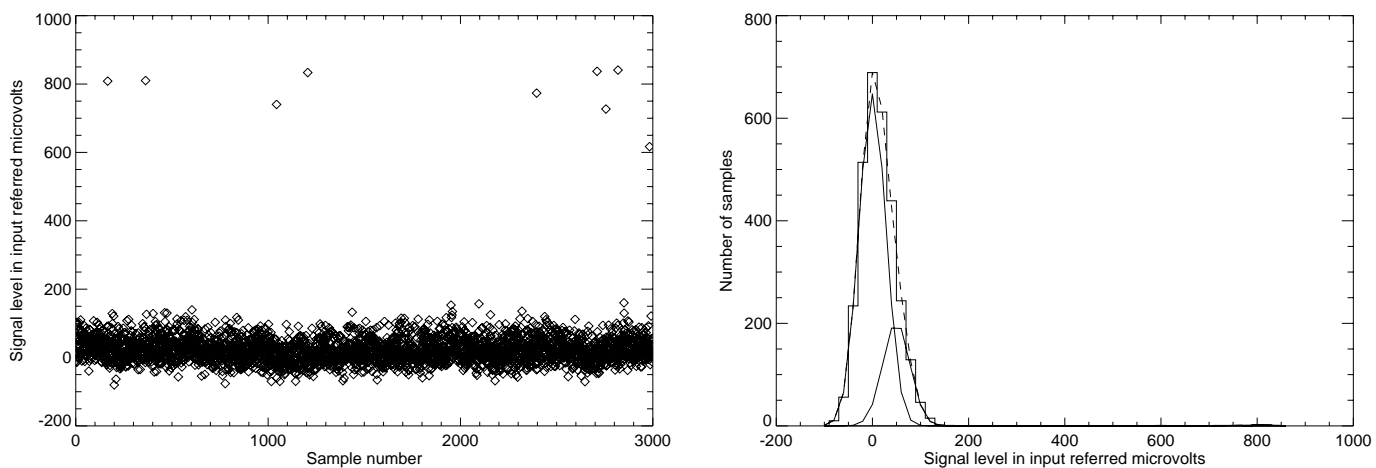

Two pixels, exhibiting $\mathrm{M}-\mathrm{U}$ and $\mathrm{S}-\mathrm{U}$ respectively with larger, easily detectable transitions are shown in 
Figures 5 and 6 . Figure 5 does not show a clear second level. However, in this case, more than one transition to approximately the same level enables burst noise detection. The samples that are not at the dominant average signal level may be mid-transition, or this may indicate multiple unstable levels. In addition, the wider Gaussian on the dominant signal level in Figure 5 could indicate burst noise on an undetectable level, similar to the detectable burst noise in the dominant signal level of Figure 6.

Though one or more unstable levels is by far the most common variation of burst noise that has been detected in the HAWAII-1RG pixels, there are definitely cases where each level is at least metastable. This leaves the last three variations, M-M, S-M, and S-S. Although they appear to be the least common, they are the variations that traditional burst noise detection algorithms detect. ${ }^{9}$ Similar to the U-U variation, a pixel can show a preference for one level over the other, or have no preferred level. One example of the M-M variation is shown in Figure 7. (Figure 2 also shows the M-M variation, but is not above the threshold of detectability by the Rayleigh criterion in our algorithm.) In Figure 7, one of the metastable states is much less preferred than the other, but it still exhibits the less preferred state for a significant amount of time before returning to the dominant state. The S-M variation can be seen in Figures 8 and 9.

Figure 7. Signal vs. sample number and corresponding histogram for a pixel exhibiting M-M burst noise.
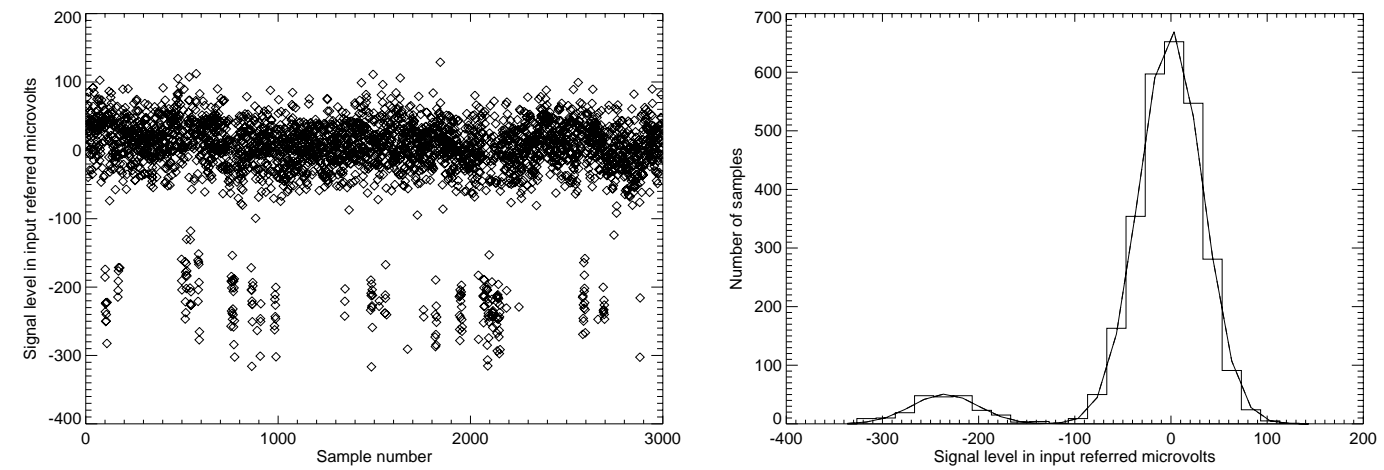

Figure 8. Signal vs. sample number and corresponding histogram for a pixel exhibiting S-M burst noise. Note the larger scale.
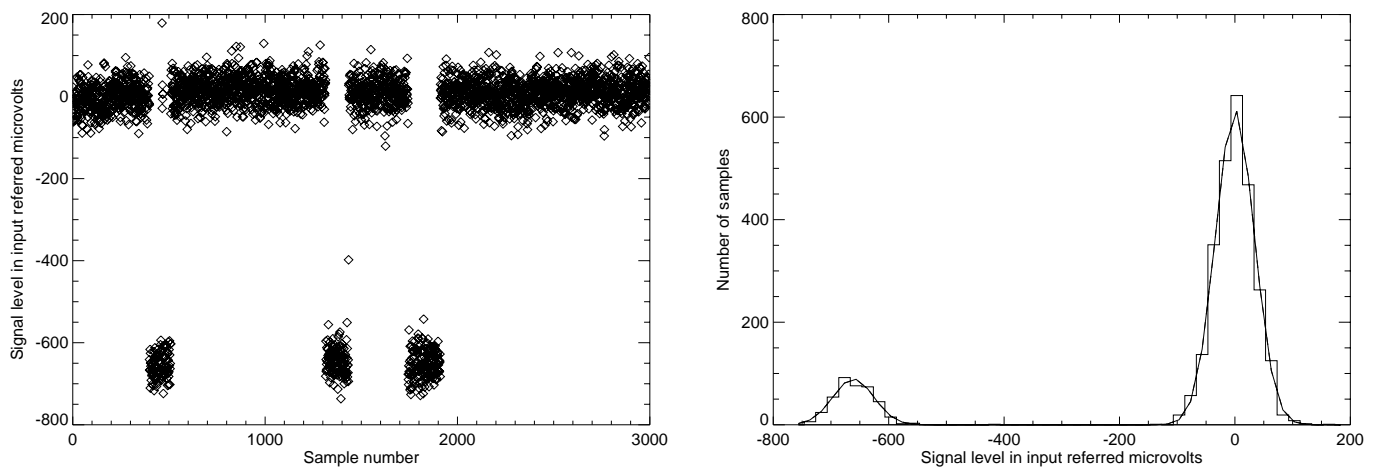

On a much slower timescale we have the least common of all variations, the S-S variation. Since these pixels can exhibit one transition in over an hour and a half integration, it is very likely that many of these pixels are overlooked in a single integration. In fact, Kandiah et al. (1989) reports observing transitions with a characteristic time spent in a single level of 20 hours. Two examples of this variation are shown in Figures 10 and 11. 
Figure 9. Signal vs. sample number and corresponding histogram for a pixel exhibiting S-M burst noise. Note the larger scale.
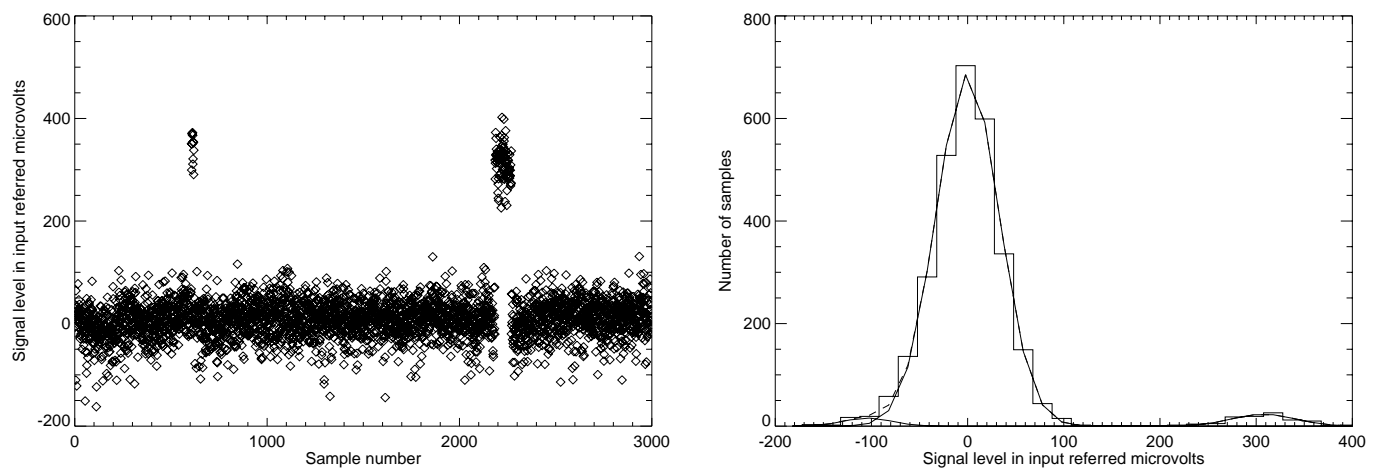

Figure 10. Signal vs. sample number and corresponding histogram for a pixel exhibiting S-S burst noise. The dashed line shows the sum of the Gaussians.
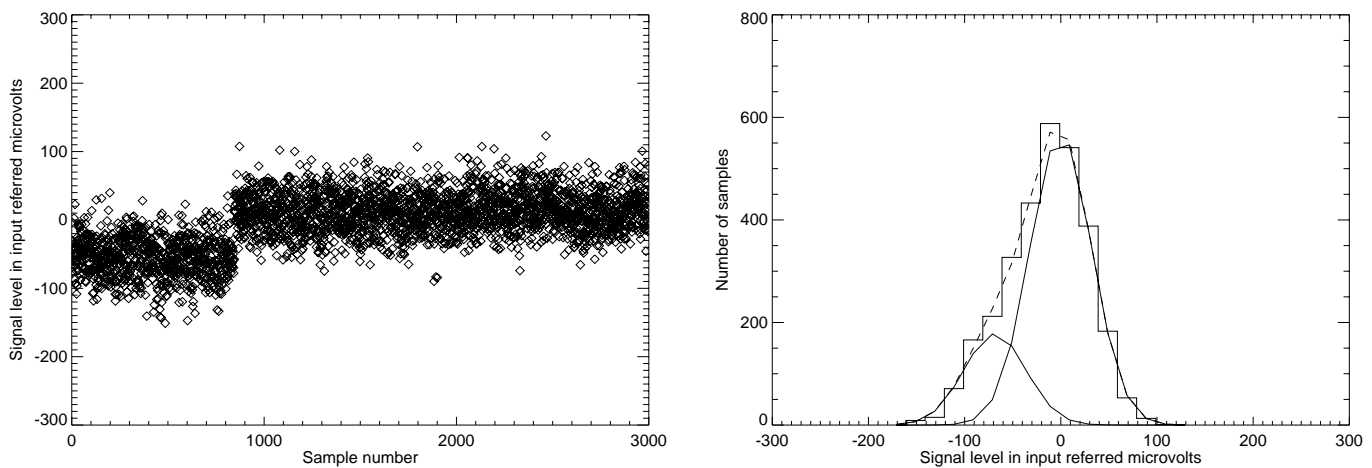

Figure 11. Signal vs. sample number and corresponding histogram for a pixel exhibiting S-S burst noise. Note the larger scale.
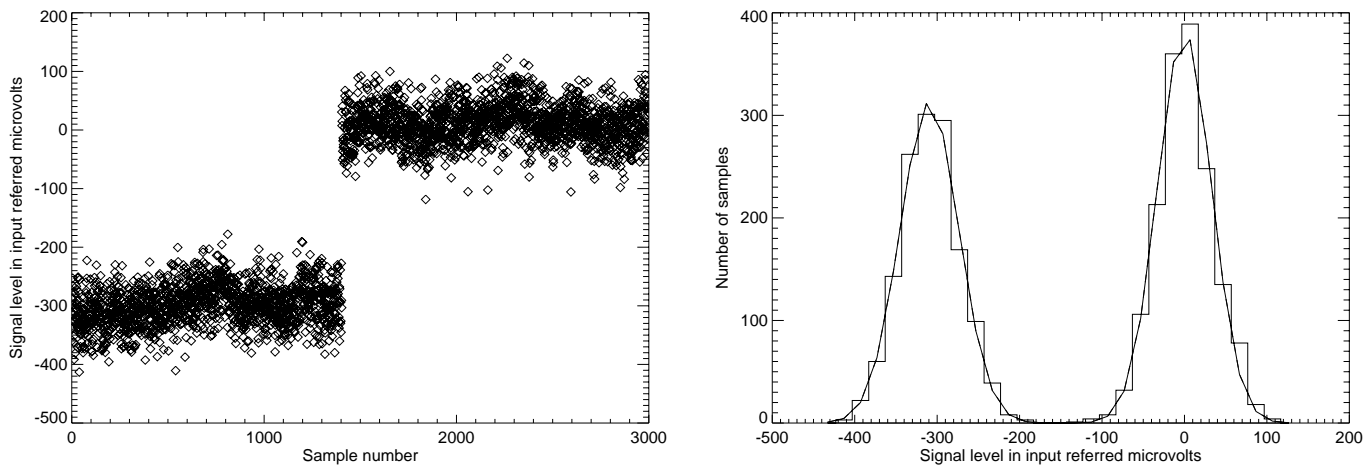

In less common circumstances (less than twenty percent of pixels identified with burst noise), a pixel may be detected with more than one additional level or many indistinguishable levels. Some examples of this are shown 
in Figures 12, 13, 14, and 15 as well as Figures 5 and 6.

Figure 12. Signal vs. sample number and corresponding histogram for a pixel exhibiting multiple level burst noise with S-S superimposed upon U-U. The dashed line shows the sum of the Gaussians.
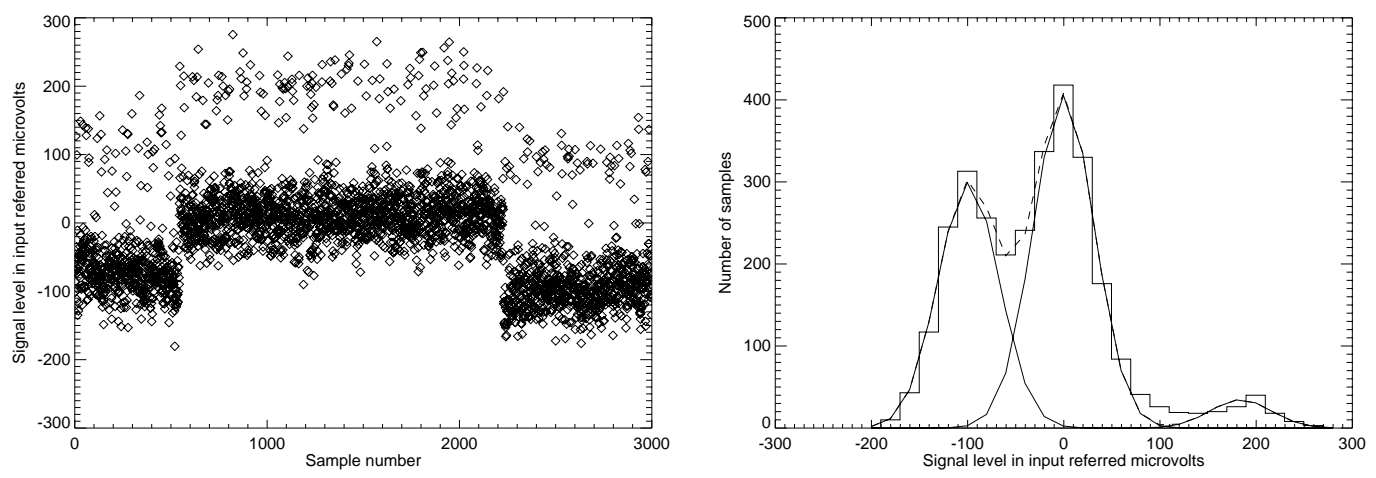

Figure 13. Signal vs. sample number and corresponding histogram for a pixel exhibiting multiple level burst noise with two defined levels and potentially many undefined levels inbetween. Note the much larger scale.
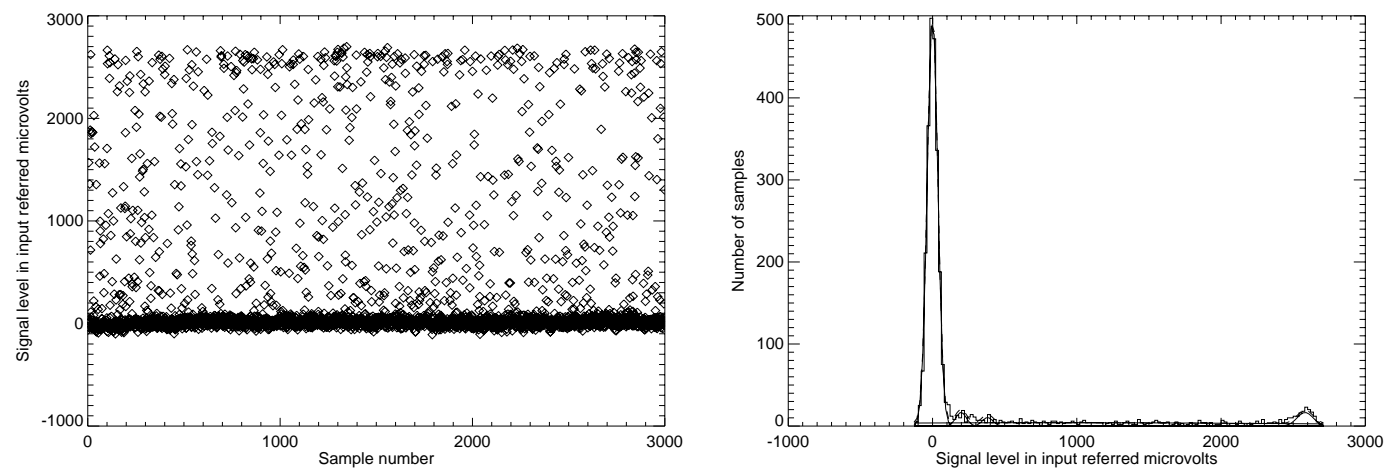

Figure 14. Signal vs. sample number and corresponding histogram for a pixel exhibiting multiple level burst noise with upper and lower secondary levels. Note the larger scale. The dashed line shows the sum of the Gaussians.
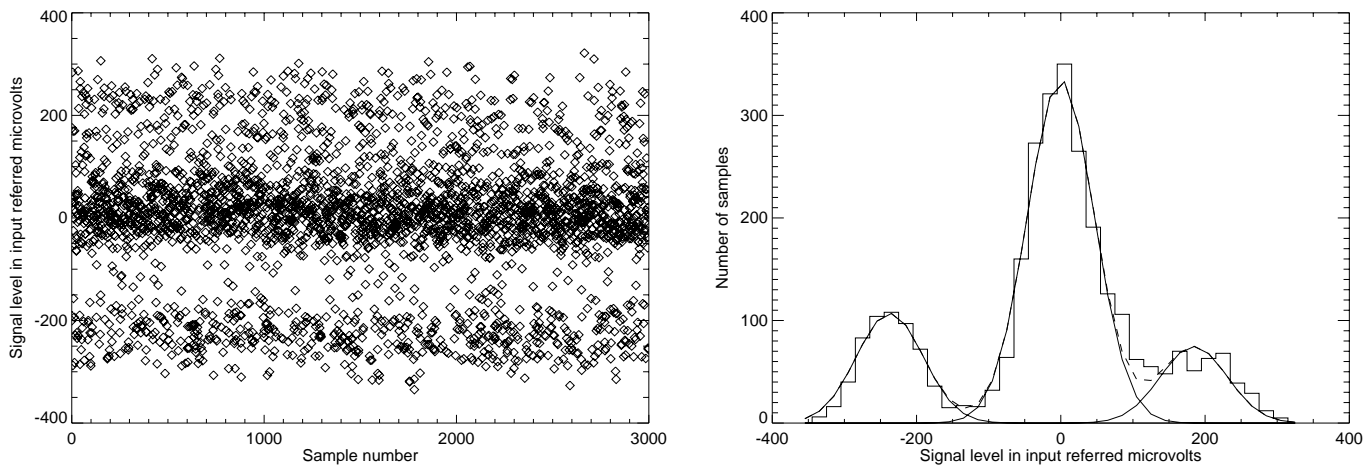
Figure 15. Signal vs. sample number and corresponding histogram for a pixel exhibiting multiple level burst noise. The dashed line shows the sum of the Gaussians. The top two figures are scaled to show the entire range of transition. The bottom two figures show the dominant level. There are many mechanisms involved which result in these various transitions.
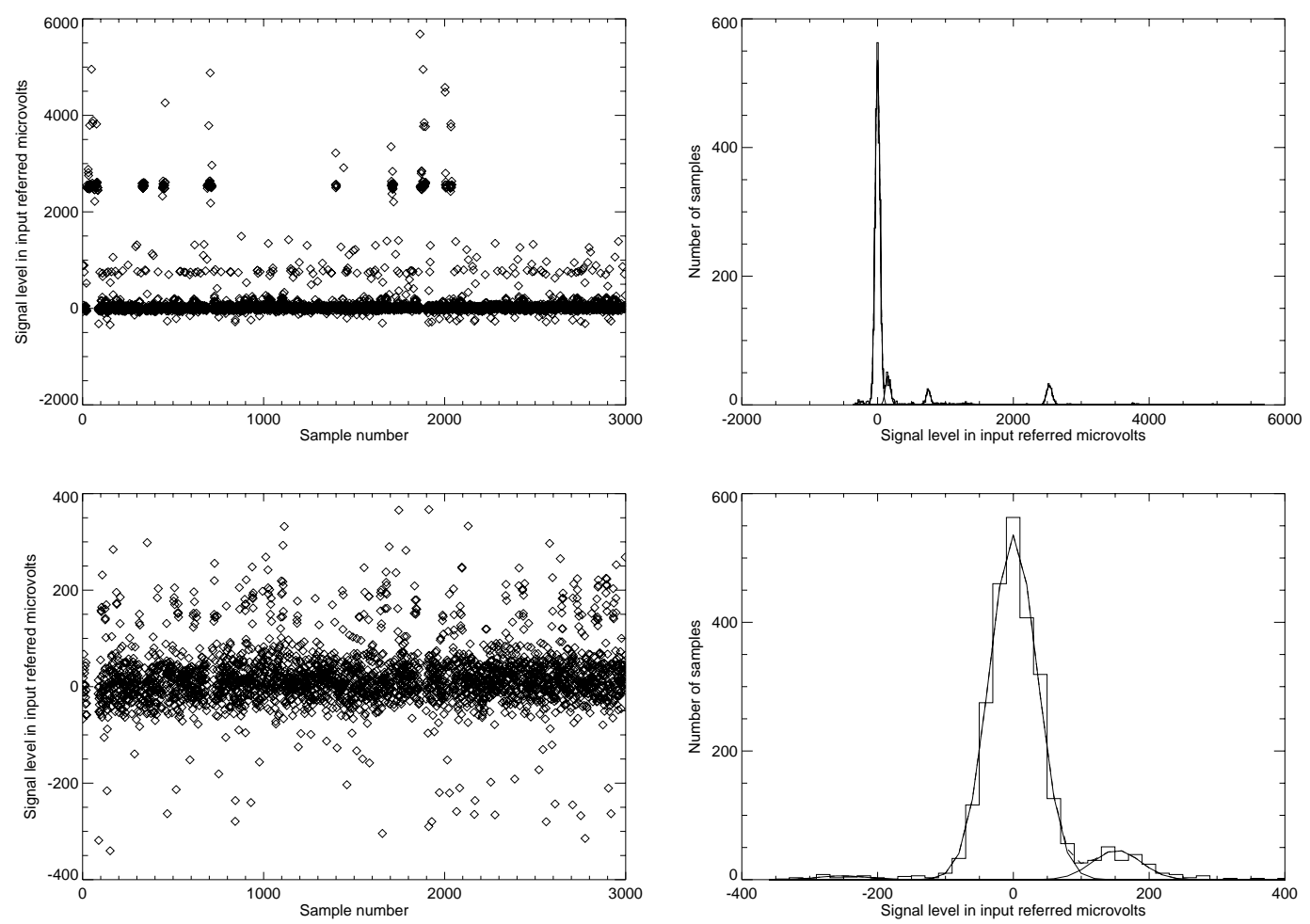

\section{RESULTS}

All of the different variations seen in Section 3 contribute to the total percentage of pixels affected by burst noise. This percentage was measured as a function of temperature using the resolution criteria given in Section 2, and the largest detected 'peak to peak' transition for each temperature is given in Table 2. These percentages do not change if the electronics are on or off during cooldown, and similar percentages were found for pixels which were and were not connected to the $\mathrm{HgCdTe}$ material.

For $37 \mathrm{~K}$ data, there are only 2000 samples used in data reduction because of a significant non-global drift that occurred in the first 1000 seconds which created false positives. Percentages computed using only the last 2000 samples are given for $30 \mathrm{~K}$ and $77 \mathrm{~K}$ for comparison. The reduction algorithm removes less significant drift exhibited by the $30 \mathrm{~K}$ and $77 \mathrm{~K}$ data.

Though the percentage of pixels exhibiting some form of detectable burst noise remains approximately the same for all three temperatures, the largest detected transition decreases with increasing temperature. In addition, the percentage of pixels identified with burst noise that exhibit three or more distinct levels decreases with increasing temperature. However, pixels that were detected with burst noise at one temperature were not the same as those detected at other temperatures, see Table 3. In fact, some pixels which exhibit one burst noise variation at $30 \mathrm{~K}$ may exhibit a totally different variation or no detectable burst noise at all at $77 \mathrm{~K}$, leading to a larger percentage of pixels containing burst noise mechanisms than are being detected at any one temperature. When tested more than once at the same temperature, though, pixels will consistently exhibit the same behavior. 
Table 2. Percentages of pixels exhibiting burst noise at various temperatures.

\begin{tabular}{cccc}
$\begin{array}{c}\text { Temperature } \\
(\mathrm{K})\end{array}$ & $\begin{array}{c}\text { Number of } \\
\text { samples used }\end{array}$ & $\begin{array}{c}\text { Percentage } \\
\text { of Pixels }\end{array}$ & $\begin{array}{c}\text { Largest } \\
\text { Transition }\end{array}$ \\
\hline 30 & 3000 & 13.4 & $10.6 \mathrm{mV}$ \\
30 & 2000 & 11.7 & $10.5 \mathrm{mV}$ \\
\hline 37 & 2000 & 11.1 & $7.7 \mathrm{mV}$ \\
\hline 77 & 3000 & 15.2 & $2.5 \mathrm{mV}$ \\
77 & 2000 & 13.5 & $2.5 \mathrm{mV}$
\end{tabular}

Table 3. Percentages of pixels exhibiting burst noise with respect to temperature.

\begin{tabular}{ccc} 
Condition & Number of Pixels & Percentage \\
\hline All three temperatures & 5372 & $2.0 \%$ \\
Any two temperatures & 29371 & $11.2 \%$ \\
Any single temperature & 74654 & $28.5 \%$
\end{tabular}

The percentage of array pixels with detectable burst noise increases as the sensitivity of detection increases. This relation between the smallest transition size detectable and the percentage of array pixels detected with burst noise is shown in Figures 16 and 17.

Figure 16. Percentage of array pixels detected vs. smallest transition size detectable.

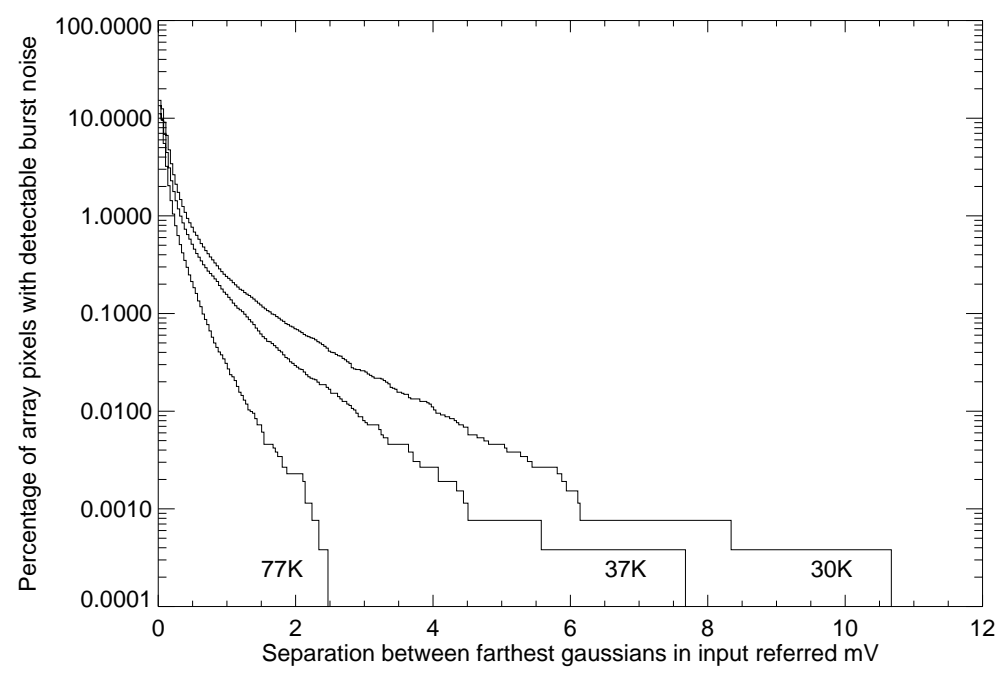


Figure 17. Percentage of array pixels detected vs. smallest transition size detectable on a log-log scale.

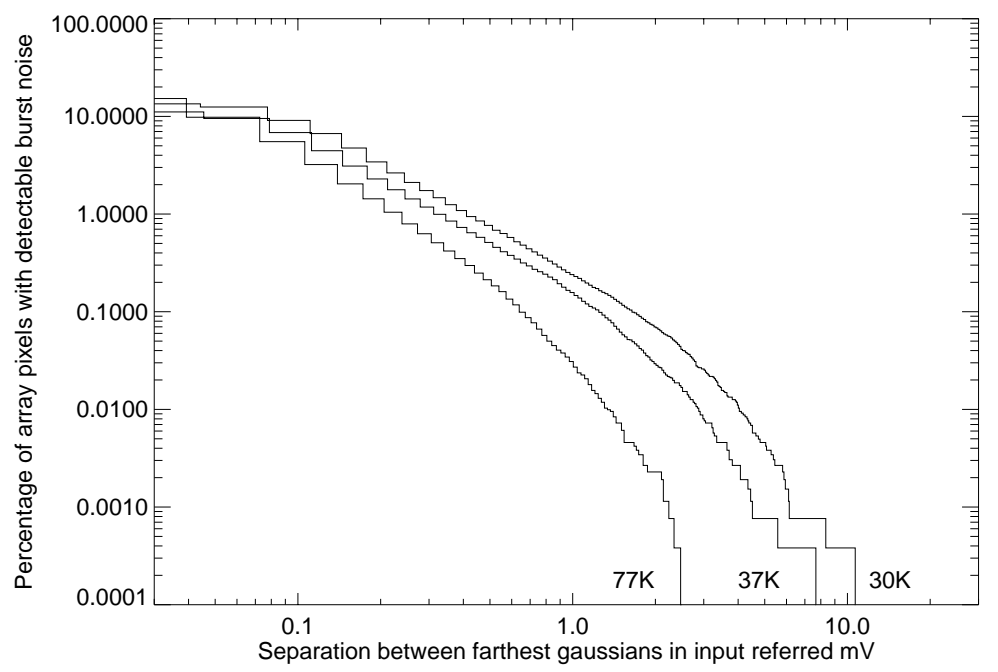

\section{CONCLUSIONS}

For the experimental situation described, we find that between 11 and $15 \%$ of pixels exhibit burst noise at a single focal plane temperature between $77 \mathrm{~K}$ and $30 \mathrm{~K}$. As noted, our burst noise detection algorithm is limited by the separation of the transition levels and the length of time between transitions. Because of this limitation, it is probable that the percentage of affected pixels is larger than we have quoted. However, for pixels with low level transition burst noise, the only discernible effect will be a small increase in total pixel noise.

Further work in this area includes finding the dependence of the timing and amplitude of the burst noise displayed by a single pixel on the source to drain current through the FET and the FET gate voltage. Comparing those results to that of other authors ${ }^{2}$ and discerning how trap characteristics correspond to observed behavior may enable us to uncover ways to compensate for this problem and/or eliminate it entirely. In conclusion, burst noise can significantly affect the pixel noise power. Therefore, elimination of the source of burst noise would make the HAWAII-1RG and -2RG even lower noise multiplexers than they already are.

\section{ACKNOWLEDGMENTS}

We are grateful for support for this program. Specifically we acknowledge NASA Ames grant NAGS2-1533. 


\section{REFERENCES}

1. S. T. Hsu and R. J. Whittier, "Physical Model for Burst Noise in Semiconductor Devices," Solid-State Electronics 13, pp. 1055-1071, 1970.

2. K. Kandiah, M. O. Deighton, and F. B. Whiting, "A physical model for random telegraph signal currents in semiconductor devices," Journal of Applied Physics 66, pp. 937-948, July 1989.

3. D. Wolf and E. Holler, "Bistable Current Fluctuations in Reverse-Biased $p$ - $n$ Junctions of Germanium," Journal of Applied Physics 38(1), pp. 189-192, 1967.

4. P. M. Campbell, E. S. Snow, W. J. Moore, O. J. Glembocki, and S. W. Kirchoefer, "Light-Activated Telegraph Noise in AlGaAs Tunnel Barriers: Optical Probing of a Single Defect," Physical Review Letters 67, pp. 1330-1333, Sept. 1991.

5. B. Dierickx and E. Simoen, "The decrease of "random telegraph signal" noise in metal-oxide-semiconductor field-effect transistors when cycled from inversion to accumulation," Journal of Applied Physics 71(4), pp. 2028-2029, 1992.

6. Y. Dai, Y. Li, and J. Xu, "Discussion on Physical Models for Burst Noise in a Forward Biased P-N Junction and Their Experimental Validation," Microelectronics Reliability 38(4), pp. 671-675, 1998.

7. J. Sikula, J. Pavelka, V. Sedlakova, M. Tacano, S. Hashiguchi, and M. Toita, "RTS in Submicron MOSFETs and Quantum Dots," Proc. SPIE, Noise and Information in Nanoelectronics, Sensors, and Standards II 5472, pp. 64-73, 2004.

8. C. M. Bacon, C. W. McMurtry, J. L. Pipher, W. J. Forrest, J. D. Garnett, D. Lee, and D. D. Edwall, "Characterization of Rockwell Scientific LWIR HgCdTe Detector Arrays," in Proc. SPIE, Focal Plane Arrays for Space Telescope, T. J. Grycewicz and C. R. McCreight, eds., 5167, pp. 313-319, Jan. 2004.

9. Bernard J. Rauscher, Donald F. Figer, Michael W. Regan, Torsten Boeker, James Garnett, Robert J. Hill, Giorgio Bagnasco, Jesus Balleza, Richard Barney, Louis E. Bergeron, Clifford Brambora, Joe Connelly, Rebecca Derro, Michael J. DiPirro, Christina Doria-Warner, Aprille Ericsson, Stuart D. Glazer, Charles Greene, Donald N. Hall, Shane Jacobson, Peter Jakobsen, Eric Johnson, Scott D. Johnson, Carolyn Krebs, Danny J. Krebs, Scott D. Lambros, Blake Likins, Sridhar Manthripragada, Robert J. Martineau, Ernie C. Morse, Samuel H. Moseley, D. Brent Mott, Theo Muench, Hongwoo Park, Susan Parker, Elizabeth J. Polidan, Robert Rashford, Kamdin Shakoorzadeh, Rajeev Sharma, Paolo Strada, Augustyn Waczynski, Yiting Wen, Selmer Wong, John Yagelowich, and Monica Zuray, "Detectors for the James Webb Space Telescope Near-Infrared Spectrograph," Proc. SPIE, Optical, Infrared, and Millimeter Space Telescopes 5487, pp. 710-726, 2004.

10. E. Simoen, B. Dierickx, C. L. Claeys, and G. J. Declerck, "Explaining the Amplitude of RTS Noise in Submicrometer MOSFETs," IEEE Transactions on Electron Devices 39(2), pp. 422-429, 1992. 